\section{How to respond}

\section{to abnormal liver}

\section{function tests}

Jane Collier MD MRCP, Consultant

Hepatologist, John Radcliffe Hospital, Oxford

Maggie Bassendine FRCP FRCP(E),

Consultant Hepatologist, Freeman Hospital,

Newcastle; Professor of Hepatology,

University of Newcastle upon Tyne

Clin Med JRCPL 2002;2:406-9

Symptoms occur only in end-stage liver disease, so many cases of liver disease are now identified in individuals incidentally found to have abnormal liver function tests (LFTs) as part of routine automated laboratory testing. Abnormal LFTs cannot be ignored because a subgroup of these patients will have progressive and potentially life-threatening liver disease for which therapeutic interventions are often available.

Even in an asymptomatic individual a careful history may identify potential

\section{Key Points}

Most chronic liver disease is identified because of an incidental finding of abnormal liver function tests (LFTs)

The commonest causes of abnormal LFTs are prescribed drugs and alcohol

Significant liver disease is unlikely if alanine aminotransferase is below $100 \mathrm{iu} / \mathrm{l}$ and a chronic liver disease screen is negative

Hepatic malignant infiltration should be considered with a very high alkaline phosphatase (ALP) in the absence of jaundice

Right hypochondrial pain in the presence of a high ALP is usually due to biliary obstruction

KEY WORDS: alcoholic liver disease, alkaline phosphatase, aminotransferases, chronic liver disease, hepatitis, liver function tests causes of abnormal LFTs (Table 1). Clues

- loss of licence due to drink driving offences

- loss of employment

- involuntary separation from partner.

History from an independent witness is also valuable where alcohol abuse is suspected.

Physical examination is often normal unless hepatomegaly is present - the finding of which lowers the threshold for investigation - or signs indicative of cirrhosis with portal hypertension (splenomegaly, ascites or spider naevi). LFTs requires an assessment of:

- pattern (hepatocellular or cholestatic)

- magnitude of elevation

- duration of abnormality

- associated symptoms

- clinical evidence of cirrhosis.

LFT abnormalities fall into several patterns which help in differentiating the underlying liver disease, indicative of:

- hepatocellular damage (high serum transaminase)

- cholestasis (high alkaline phosphatase (ALP))

- poor synthetic function.

Although a liver biopsy is the gold standard for assessing both aetiology and the degree of fibrosis, it is associated with some morbidity and should be undertaken only after assessing the riskto covert alcohol abuse include:

An appropriate response to abnormal

benefit. The clinical setting, together with the specific pattern of LFT abnormality and appropriate additional tests, can narrow the differential diagnosis and provide a cost-effective approach to identifying those patients who need a liver biopsy.

\section{Threshold for investigating liver function test abnormalities}

Abnormal LFTs in the acute hospital and/or intensive care setting are common. The cause is almost always multifactorial, including intrahepatic cholestasis from sepsis, hepatic congestion from heart failure and, less commonly, drugs. Acute changes in LFTs also occur secondary to other organ dysfunction, such as hyper- or hypothyroidism and active rheumatoid arthritis; these normalise on treatment of the underlying condition.

In an asymptomatic, non-jaundiced patient with abnormal LFTs it is worth initially:

- excluding an alcohol aetiology and reducing alcohol intake

- stopping drugs started within the last three months

- excluding drug toxicity from non-prescribed herbal remedies ${ }^{1}$

- repeating LFTs in 2-3 weeks.

If LFTs remain abnormal, an appropriate chronic liver disease screen and an abdominal ultrasound scan should be undertaken (see below).

Table 1. Clinical features that help to indicate the cause of abnormal liver function tests.

Clinical clue

Disease

Alcohol abuse (overt/covert)

Increased body mass index

Non-insulin dependent diabetes mellitus

Previous intravenous drug use

Non-sterile needle use (eg war service)

Blood transfusion prior to 1990

Recent new drugs or herbal remedies

Family history of liver disease

History of other autoimmune disease

Recent travel to developing nations

Diarrhoea
Alcoholic liver disease

Non-alcoholic steatohepatitis

Chronic hepatitis C or B

Chronic hepatitis $\mathrm{C}$

Drug-induced hepatotoxicity

Haemochromatosis

Wilson's disease

Primary biliary cirrhosis

Autoimmune hepatitis

Acute hepatitis A or $E$

Primary sclerosing cholangitis 
Hepatomegaly implies significant liver pathology and is an indication for further investigation even in the presence of mild LFT abnormality.

\section{Hepatocellular damage: raised serum transaminase}

\section{Degree of elevation}

The two commonly measured serum transaminases which act as sensitive indicators of hepatocellular damage are alanine (ALT) aminotransferase and aspartate (AST) aminotransferase ${ }^{2}$. The less specific AST is localised in organs other than the liver, including heart, skeletal muscle, kidney and brain. The magnitude of enzyme level elevation does not correlate with eventual outcome but may be a helpful indicator of possible underlying aetiology (Table 2).

ALT levels tend to be near normal in alcoholic liver disease. More often, there is a mixed picture with an elevated ALP particularly in the presence of hepatic fibrosis. Even in severe alcoholic hepatitis the ALT and AST levels rarely rise to more than seven times the upper limit of normal (ULN), and the prognosis is related to other measures of hepatic dysfunction (see below). If transaminase levels above 1,000 iu/l are found in an alcoholic, it is important to exclude coexistent pathology such as acute viral hepatitis or inadvertent paracetamol toxicity.

\section{Alanine transaminase and/or aspartate transaminase}

There are occasions when it is useful to measure both ALT and AST. An isolated elevated AST may be due to muscle damage, and the AST to ALT ratio is useful diagnostically in alcoholic liver disease because $70 \%$ of patients have a ratio greater than two.

\section{Other investigations}

If an isolated ALT or AST is persistently less than 2.5 times the ULN, chronic viral hepatitis, autoimmune hepatitis ${ }^{3}$ and haemochromatosis should be excluded (Table 3). Wilson's disease can also
Table 2. Common causes of elevated serum transaminases.

\begin{tabular}{ll} 
Elevation & Cause \\
\hline Minor $(<100 \mathrm{iu} / \mathrm{l})$ & Chronic hepatitis C \\
& Chronic hepatitis B \\
& Haemochromatosis \\
& Fatty liver \\
Moderate (100-300 iu/l) & As above plus: \\
& Alcoholic hepatitis \\
& Non-alcoholic steatohepatitis \\
& Autoimmune hepatitis \\
& Wilson's disease \\
& Drug toxicity, particularly paracetamol \\
Major $(>1,000 \mathrm{iu} / \mathrm{l})$ & Acute viral hepatitis \\
& Autoimmune hepatitis \\
& Ischaemic liver
\end{tabular}

mimic chronic hepatitis in someone below the age of 40 . If this chronic liver disease screen is negative, it is less likely that a liver biopsy will add to management.

\section{Non-alcoholic steatohepatitis}

A liver biopsy should be considered if a chronic liver screen is negative but liver biochemistry remains abnormal (ALT/AST more than twice the ULN) as up to $25 \%$ of these patients may have significant liver fibrosis ${ }^{4}$. Non-alcoholic steatohepatitis is an increasingly recognised condition associated with an

increased body mass index, diabetes mellitus and hypercholesteraemia ${ }^{5}$. Histologically, it mimics alcoholic hepatitis and may present only with elevated serum transaminases. It is associated with progression to cirrhosis, so it is important to recognise.

\section{Haemochromatosis}

If a transferrin saturation is greater than $55 \%$, haemochromatosis should be excluded by looking for a mutation in the HFE gene. Over $90 \%$ of haemochromatotics in the UK are homozygous for the $\mathrm{C} 282 \mathrm{Y}$ mutation and a smaller

Table 3. Chronic liver disease screening tests

Investigation

Hepatitis C antibody
HCV RNA (as $20 \%$ clear virus)
Hepatitis B surface antigen
Transferrin saturation ( $>55 \%$ )
HFE genotype
Antimitochondrial antibodies
Anti-smooth muscle antibody
Anti-nuclear antibodies
Immunoglobulins:
IgG
IgM
IgA
$\alpha 1-$ antitrypsin (level and phenotype)
Low caeruloplasmin*
(genetic test not routinely used)

*This may mimic autoimmune hepatitis and should be considered in a patient below 40 years old with elevated alanine aminotransferase. Diagnosis is confirmed by ophthalmic finding of Kayser-Fleischer rings and high dry weight liver copper in the absence of cholestasis.

$\mathrm{HCV}=$ hepatitis $\mathrm{C}$ virus 
percentage are compound heterozygotes (C282Y/H63D). A liver biopsy is not necessary to establish the degree of fibrosis if the ferritin is below 1,000 iu/l as structural liver disease is unusual with this degree of iron overload ${ }^{6}$.

\section{Cholestasis: elevated alkaline phosphatase}

\section{Associated symptoms}

ALP is produced by bile ducts but also by bone, intestine and placenta. The most common cause of an elevated ALP is biliary obstruction, most likely if pain is present. In contrast, the presence of itching in the absence of jaundice suggests intrahepatic disease (ie primary biliary cirrhosis (PBC) or primary sclerosing cholangitis (PSC)) (Table 4). Secondary cholangitis, due to biliary obstruction, a blocked biliary stent or PSC, will cause worsening of liver biochemistry which improves on treating the infection. In the elderly, biliary obstruction from common bile duct stones may present only with confusion and an elevated ALP.

\section{Degree of elevation}

Very high ALP levels ( $>1,000 \mathrm{iu} / \mathrm{l}$, particularly if $>2,000 \mathrm{iu} / \mathrm{l}$ ) in the absence of jaundice is indicative of hepatic infiltration. It is important to exclude malignant infiltration particularly if there is weight loss. Low ALP levels have been described in patients with acute onset of Wilson's disease.

\section{Other investigations}

All patients with cholestatic LFTs should undergo ultrasound scanning to exclude biliary obstruction and liver metastases. Even if the bile ducts are not dilated on ultrasound, imaging of the biliary tree is indicated in the presence of cholangitis or pain to exclude common bile duct stones. If the ultrasound is normal, PBC should be excluded by an antimitochondrial (AMA) antibody specific for $\mathrm{PBC}^{7}$. A liver biopsy should be considered if the ALP is more than twice the ULN in the presence of a normal ultrasound and negative AMA. An endoscopic retrograde cholangiopancreatography (ERCP) is needed to exclude PSC because a liver biopsy may not be diagnostic; this should be considered, especially if there is a history of colitis.

Large duct obstruction can be excluded non-invasively by magnetic resonance cholangiopancreatography, but resolution is not good enough to exclude abnormalities of the finer intrahepatic ducts so ERCP probably still has a diagnostic role in excluding PSC.

\section{Gamma-glutamyl transferase}

The main use of gamma glutamyl transferase (GGT), which is also produced by bile ducts, is in differentiating bone from liver ALP. GGT is elevated both in chronic liver disease (ie cirrhosis), even in the absence of active alcohol misuse and so is not diagnostic of current alcohol misuse - and in uncomplicated hepatic steatosis. Induction of the enzyme also occurs due to many drugs including chlorpromazine and phenytoin. An isolated raised GGT in an individual with a normal prothrombin time (PT) and albumin in the absence of clinical signs of liver disease does not need further investigation.

\section{Tests of hepatic dysfunction}

The tests discussed above are often termed 'liver function tests', but the term is a misnomer. Tests to measure the synthesis by the liver of proteins (eg albumin, clotting factors) or its capacity to metabolise drugs (eg galactose clearance) can more accurately be termed LFTs. However, the latter are rarely used in routine practice.

\section{Albumin}

Measurement of serum albumin is useful in chronic liver disease and is a component of grading systems (see below). Obviously, non-hepatic causes of hypoalbuminaemia need to be excluded (eg proteinuria, malnutrition). Albumin has a long half-life (20-30 days) and is not therefore a good indicator of liver dysfunction in acute hepatic injury.

\section{Prothrombin time}

PT is a useful indicator of liver failure in both acute and chronic hepatic injury, provided that cholestasis with malabsorption of vitamin $\mathrm{K}$ has been excluded by parenteral administration of vitamin $\mathrm{K}$ (10 mg/day for three days). In acute liver failure, the PT may rise to over 50 seconds and is the most important predictor of outcome. Specialist advice from a liver transplant centre should be sought early. It should be remembered that there are large inter-laboratory differences in PT results due to variability in thromboplastin reagents used in the assay. In cirrhosis, levels are usually two to five seconds prolonged. A PT elevated to over 20 seconds in a cirrhotic is an indication of very poor synthetic function and is associated with a poor outcome (see below).

Table 4. Common causes of elevated alkaline phosphatase.

Cause

Large duct biliary obstruction

Primary biliary cirrhosis (common in women)

Primary sclerosing cholangitis

Intrahepatic cholestasis of sepsis

Drugs

Infiltration of liver

\section{Common bile duct stones \\ Chronic pancreatitis \\ Carcinoma of pancreas \\ Cholangiocarcinoma}

Co-proxamol

Co-amoxiclav

Sarcoid/amyloid

Granulomatous hepatitis

Liver metastases 


\section{Bilirubin}

Bilirubin is conjugated in the liver, and $90 \%$ of serum bilirubin will be conjugated. A rise in bilirubin is a reflection of the severity of liver damage, parenchymal or biliary. An elevated bilirubin is thus an adverse prognostic marker in both alcoholic hepatitis and end-stage PBC.

An isolated elevated bilirubin is usually due to elevated unconjugated bilirubin. The most common cause is Gilbert's syndrome, a benign condition in which a genetic mutation present in $\mathbf{s} 13 \%$ of Whites causes impaired bilirubin glucuronidation. It does not require further investigation, but it is important to exclude haemolysis which is suggested by a low haemoglobin, elevated reticulocyte count and splenomegaly.

\section{Prognostic scoring}

The above tests of hepatic dysfunction are common components of prognostic scoring systems. For example, in a patient with severe acute alcoholic hepatitis, a discriminant function greater than 32, calculated using the formula 4.6 ( PT - control time $)+$ serum bilirubin (in $\mu \mathrm{mol} / \mathrm{l}$ )/17.1, should be considered for corticosteroid therapy ${ }^{8}$ and referral to a liver centre. In cirrhosis, the prognosis of patients can be assessed using the modified Child-Pugh classification (Table 5), which incorporates bilirubin, albumin and PT levels.

\section{Biochemical evidence of cirrhosis and hepatic fibrosis}

In the absence of signs of portal hypertension (splenomegaly and ascites), liver enzyme abnormalities associated with biochemical evidence of hepatic dysfunction (elevated bilirubin, low serum albumin, prolonged PT) suggest that disease is advanced. However, patients with cirrhosis may have entirely normal or near normal transaminase levels. A patient with Child-Pugh grade $\mathrm{B}$ or $\mathrm{C}$ cirrhosis under 65 years should be considered for referral to a liver transplant centre. It is usually recommended

Table 5. Modified Child-Pugh prognostic classification for grading hepatic dysfunction in patients with cirrhosis.

Points*

\begin{tabular}{lccc}
\hline Factor & 1 & 2 & 3 \\
\hline Encephalopathy & 0 & $1-2$ & $3-4$ \\
Ascites & None & Slight & Moderate \\
Bilirubin $(\mu \mathrm{mol} / \mathrm{l})$ & $<34$ & $34-50$ & $>50$ \\
Albumin $(\mathrm{g} / \mathrm{l})$ & $<35$ & $28-35$ & $<28$ \\
PT (control time) (sec) & $1-4$ & $5-6$ & $>6$ \\
\hline
\end{tabular}

* Total points: 5 or $6=$ grade $A ; 7-9=$ grade $B ; 10-15$ grade $C$.

PT $=$ prothrombin time.

that patients with Child-Pugh grade A cirrhosis are screened for the development of hepatocellular cancer with six-monthly liver ultrasound scans and serum $\boldsymbol{\alpha}$-fetoprotein levels?

\section{Serum markers of hepatic fibrosis}

There is great interest in developing serum markers of hepatic fibrosis, to avoid the need for a liver biopsy to assess the degree of hepatic fibrosis in patients in whom the cause of liver dysfunction has been identified (eg chronic hepatitis $\mathrm{C}$ infection). In psoriasis, procollagen peptide type 3 shows some correlation with hepatic fibrosis due to methotrexate but does not have a sufficiently high predictive value to use in other liver disease ${ }^{10}$. However, a recent study has suggested that hepatic fibrosis can be predicted in chronic hepatitis $\mathrm{C}$ infection using a combination of $\boldsymbol{\alpha} 2$-macroglobulin, haptoglobulin, gamma globulin, apolipoprotein A1 and GGT levels ${ }^{11}$. Several other candidate markers of fibrogenesis are likely to emerge over the next few years.

\section{References}

1 Aithal GP, Rawlins MD, Day CP. Clinical diagnostic scale: a useful tool in the evaluation of suspected hepatotoxic adverse drug reactions. J Hepatol 2000;33:949-52.

2 Kew MC. Serum aminotransferase concentration as evidence of hepatocellular damage. Lancet 2000;355:591-2.

3 Alvarez F, Berg PA, Bianchi FB, Bianchi L et al. International Autoimmune Hepatitis Group Report: review of criteria for diag- nosis of autoimmune hepatitis. J Hepatol 1999;31:929-38.

4 Skelly MM, James PD, Ryder SD. Findings on liver biopsy to investigate abnormal liver function tests in the absence of diagnostic serology. J Hepatol 2001;35:195-9.

5 Marchesini G, Forlani G. NASH: from liver diseases to metabolic disorders and back to clinical hepatology. Hepatology 2002;35: 497-9.

6 Guyader D, Jacquelinet C, Moirand R, Turlin B et al. Noninvasive prediction of fibrosis in C282Y homozygous haemochromatosis. Gastroenterology 1998;115:929-36.

7 Jones DE, James OF, Bassendine MF. Primary biliary cirrhosis - clinical and associated autoimmune features and natural history. Clin Liver Dis 1998;2:265-82.

8 Maddrey WC. Alcohol-induced liver disease. Review. Clin Liver Dis 2000;4:115-31.

9 Bruix J, Sherman M, Llovet JM, Beaugrand $\mathrm{M}$ et al. Clinical management of hepatocellular carcinoma. Conclusions of the Barcelona-2000 EASL conference. European Association for the Study of the Liver. J Hepatol 2001;35:421-30.

10 Zachariae H, Heickendorff L, Sogaard H. The value of amino-terminal propeptide of type III procollagen in routine screening for methotrexate-induced liver fibrosis: a 10-year follow-up. Br J Dermatol 2001;144: $100-3$.

11 Imbert-Bismut F, Ratziu V, Pieroni L, Charlotte $\mathrm{F}$ et al. Biochemical markers of liver fibrosis in patients with hepatitis $\mathrm{C}$ virus infection: a prospective study. Lancet 2001;357:1069-75. 